\title{
Methods and models of protecting computer networks from un- wanted network traffic
}

\author{
Gulomov Sherzod Rajaboevich $^{1}{ }^{*}$, Ganiev Abdukhalil Abdujalilovich ${ }^{1}$ \\ ${ }^{1}$ Providing Information Security Department, Tashkent University of Information Technologies named after Muhammad al-Khwarizmi, \\ Uzbekistan \\ *Corresponding author E-mail: sherhisor30@gmail.com
}

\begin{abstract}
In this article a method of measure network traffic to collect data about the header of packets and to analyze the traffic dump in computer networks are offered. A method for detecting anomalies and a formal model for protecting information from DDoS attacks, which make it possible to simplify the development of filter rule sets and improve the efficiency of computer networks, taking into account, the interaction of detection modules and the use of formal set-theoretic constructions are proposed.
\end{abstract}

Keywords: TCP SYN Flood; Ping of Death; Tribe Flood Network (TFN); Stacheldraht; IP Spoofing.

\section{Introduction}

Currently, the basic concepts of cyber security are accessibility, integrity and confidentiality. Distributed Denial of Service (DDoS) attacks affects the availability of information resources. DDoS is considered successful if it has led to inaccessibility of the information resource. The success of the attack and the impact on the target resources are different in that the impact damages the victim.

There are several methods for increasing the power of DDoS attacks, but the basic idea is almost the same. The attacker performs IP spoofing and sends fake requests to the vulnerable UDP-server. Not knowing that the requests are fakes, the server is preparing a response. The problem occurs when the server sends thousands of replies to the attacked host, thereby causing its denial of service. Attacks using the enhancement methods are very effective, since the size of the response packets exceeds the size of the request packets. As a result, an attacker, even with insignificant resources, can implement a powerful DDoS attack. Researchers regularly record such attacks, but new previously unknown methods, cybercriminals use extremely rarely. This includes, in particular, the Memcrashed attack, which involves augmenting the attack using memcached UDP. In recent days, the number of attacks Memcrashed began to grow rapidly.

\section{Hierarchy of processes for measuring net- work traffic}

The measurement of network traffic consists of two phases: data acquisition and analysis.

At the first phase, a procedure is performed for the direct measurement of traffic characteristics. Data collection is carried out at any point of the network by tools that register packet traffic, with information on the packet headers and the time stamp for their registration.
At the second phase, the processing of the collected information is carried out using tools that allow you to convert data according to the models used and provide traffic characteristics in the form of numerical values or initial data for plotting.

Tools, used during the measurement: a utility for capturing IP packets on the network interface - creating a traffic dump, a dump analysis utility, a graphing utility.

The measurement objects can be physical signals in a transmission channel, individual packets, and virtual connections consisting of sequence packets and aggregated traffic [1]. When taking measurements, the following important factors should be considered:

- Each dimension changes the state of the packet. When a packet passes through an intermediate network device, the Time to Live (TTL) is reduced by one and the checksum is recalculated for each packet;

- Packet measurement "disturbs the network". There is an addition of delays in the process of transferring packets, which in turn leads to the appearance of new properties;

- Measured traffic characteristics can be obtained on the time interval that is associated with the minimum packet size and the lifetime of the virtual TCP connection.

To estimate the measurements of network traffic, it used the throughput of the network of the Tashkent University of Information Technologies named after Muhammad al-Khwarizmi and the length of the packet. In the results of the experiment, it was determined that the throughput of the network of the Tashkent University of Information Technologies named after Muhammad al-Khwarizmi is

$100 \mathrm{Mbit} / \mathrm{s}$. Estimates of network traffic measurements are denoted by the formula:

$\Delta \mathrm{t}=\frac{\mathrm{l}_{\mathrm{p}}}{\lambda}$

Where

$\mathrm{l}_{\mathrm{p}}$ - Length of the packet;

$\lambda$ - Channel throughput. 
In Table 1 is shown the results of estimates of network traffic the packets. measurements in different channel throughput and the length of

Table 1: Estimates of Network Traffic Measurements in Different Channel Throughput and Length of the Packet

\begin{tabular}{|c|c|c|c|c|c|c|c|}
\hline № & $\begin{array}{l}\text { length of the } \\
\text { packet } l_{p} \\
\text { (minimum) }\end{array}$ & $\begin{array}{l}\text { length of } \\
\text { the } \\
\text { packet } l_{p} \\
\text { (long) }\end{array}$ & $\begin{array}{l}\text { length of } \\
\text { the } \\
\text { packet } l_{p} \\
\text { (higher } \\
\text { long) }\end{array}$ & $\begin{array}{l}\text { Throughput } \\
\lambda\end{array}$ & $\begin{array}{l}\text { Estimates of network } \\
\text { traffic measurements for } \\
\text { the minimum packet } \\
\Delta \mathrm{t}=\frac{\mathrm{l}_{\mathrm{p}}}{\lambda}\end{array}$ & $\begin{array}{l}\text { Estimates of network } \\
\text { traffic measurements } \\
\text { for a long packet } \\
\Delta t=\frac{l_{p}}{\lambda}\end{array}$ & $\begin{array}{l}\text { Estimates of network } \\
\text { traffic measurements for } \\
\text { a higher long packet } \\
\Delta \mathrm{t}=\frac{\mathrm{l}_{\mathrm{p}}}{\lambda}\end{array}$ \\
\hline 1 & $\begin{array}{l}64 \text { bytes }= \\
0.00048 \text { Mbit }\end{array}$ & $\begin{array}{l}1500 \text { bytes } \\
=0.011 \\
\text { Mbit }\end{array}$ & $\begin{array}{l}6000 \text { bytes } \\
=0.045 \\
\text { Mbit }\end{array}$ & $10 \mathrm{Mbit} / \mathrm{s}$ & $\begin{array}{l}0,000048=48 \times \\
10^{-6} \mathrm{Mbit} / \mathrm{s}\end{array}$ & $\begin{array}{l}0,0011=11 \times \\
10^{-4} \mathrm{Mbit} / \mathrm{s}\end{array}$ & $\begin{array}{l}00,0045=45 \\
\times 10^{-4} \mathrm{Mbit} / \mathrm{s}\end{array}$ \\
\hline 2 & $\begin{array}{l}64 \text { bytes }= \\
0.00048 \text { Mbit }\end{array}$ & $\begin{array}{l}1500 \text { bytes } \\
=0.011 \\
\text { Mbit }\end{array}$ & $\begin{array}{l}6000 \text { bytes } \\
=0.045 \\
\text { Mbit }\end{array}$ & $50 \mathrm{Mbit} / \mathrm{s}$ & $\begin{array}{l}0.0000096=96 \times 10^{-7} \\
\mathrm{Mbit} / \mathrm{s}\end{array}$ & $\begin{array}{l}0,00022=22 \times 10^{-5} \\
\mathrm{Mbit} / \mathrm{s}\end{array}$ & $0,0009=9 \times 10^{-4} \mathrm{Mbit} / \mathrm{s}$ \\
\hline 3 & $\begin{array}{l}64 \text { bytes }= \\
0.00048 \text { Mbit }\end{array}$ & $\begin{array}{l}1500 \text { bytes } \\
=0.011 \\
\text { Mbit }\end{array}$ & $\begin{array}{l}6000 \text { bytes } \\
=0.045 \\
\text { Mbit }\end{array}$ & $100 \mathrm{Mbit} / \mathrm{s}$ & $\begin{array}{l}0.0000048=48 \times 10^{-7} \\
\mathrm{Mbit} / \mathrm{s}\end{array}$ & $\begin{array}{l}0.00011=11 \times 10^{-5} \\
\text { Mbit/s }\end{array}$ & $\begin{array}{l}0,00045=45 \times \\
10^{-5} \mathrm{Mbit} / \mathrm{s}\end{array}$ \\
\hline 4 & $\begin{array}{l}64 \text { bytes }= \\
0.00048 \text { Mbit }\end{array}$ & $\begin{array}{l}1500 \text { bytes } \\
=0.011 \\
\text { Mbit }\end{array}$ & $\begin{array}{l}6000 \text { bytes } \\
=0.045 \\
\text { Mbit }\end{array}$ & $1000 \mathrm{Mbit} / \mathrm{s}$ & $\begin{array}{l}0.00000048=48 \times 10^{-8} \\
\mathrm{Mbit} / \mathrm{s}\end{array}$ & $\begin{array}{l}0.000011=11 \times 10^{-6} \\
\mathrm{Mbit} / \mathrm{s}\end{array}$ & $\begin{array}{l}0,000045=45 \times \\
10^{-6} \mathrm{Mbit} / \mathrm{s}\end{array}$ \\
\hline 5 & $\begin{array}{l}64 \text { bytes }= \\
0.00048 \text { Mbit }\end{array}$ & $\begin{array}{l}1500 \text { bytes } \\
=0.011 \\
\text { Mbit }\end{array}$ & $\begin{array}{l}6000 \text { bytes } \\
=0.045 \\
\text { Mbit }\end{array}$ & $5000 \mathrm{Mbit} / \mathrm{s}$ & $\begin{array}{l}0.000000096=96 \times 10^{-9} \\
\mathrm{Mbit} / \mathrm{s}\end{array}$ & $\begin{array}{l}0.0000022=22 \times 10^{-7} \\
\text { Mbps }\end{array}$ & $0.000009=9 \times 10^{-6} \mathrm{Mbit} / \mathrm{s}$ \\
\hline 6 & $\begin{array}{l}64 \text { bytes }= \\
0.00048 \text { Mbit }\end{array}$ & $\begin{array}{l}1500 \text { bytes } \\
=0.011 \\
\text { Mbit }\end{array}$ & $\begin{array}{l}6000 \text { bytes } \\
=0.045 \\
\text { Mbit }\end{array}$ & $\begin{array}{l}10,000 \\
\text { Mbit/s }\end{array}$ & $\begin{array}{l}0.000000048=48 \times 10^{-9} \\
\mathrm{Mbit} / \mathrm{s}\end{array}$ & $\begin{array}{l}0.0000011=11 \times 10^{-7} \\
\mathrm{Mbit} / \mathrm{s}\end{array}$ & $\begin{array}{l}0,0000045= \\
45 \times 10^{-7} \mathrm{Mbit} / \mathrm{s}\end{array}$ \\
\hline
\end{tabular}

It should be noted that measuring the characteristics of network traffic makes it possible to collect information about packet headers and provide a packet in the form of numeric values, which allows to detect packets and analyze the traffic dump at any point on the network.

\section{Method for detecting anomalies in the net- work traffic}

Causes and sources of anomalies in the network traffic. There are visible anomalies, manifested in the incorrect operation of the system at the current time and anomalies that have no visible signs at the current time, but which can lead to failures after a considerable time. At the same time, the more dangerous are the anomalies that arise as a result of the DoS attack.

In Table 2 is described the various and consequences of anomalies in network traffic.

Table 2: The Causes and Consequences of Anomalies in the Network Traffic

\begin{tabular}{|c|c|c|}
\hline $\begin{array}{l}\text { The cause of the } \\
\text { anomalies }\end{array}$ & Type of manifestation of anomalies & Consequences \\
\hline $\begin{array}{l}\text { Application-level } \\
\text { attacks }\end{array}$ & $\begin{array}{l}\text { Exploitation of known vulnerabilities and errors in the } \\
\text { software, scanning and access to ports associated with } \\
\text { vulnerable applications. }\end{array}$ & $\begin{array}{l}\text { Attackers can access the network, increase privileges, and gain } \\
\text { administrative access. }\end{array}$ \\
\hline Auto router & Traffic jumps on flows & $\begin{array}{l}\text { Installing rootkit and using the system to automate the intrusion } \\
\text { process, allows an attacker to scan hundreds of thousands of } \\
\text { systems in a short time }\end{array}$ \\
\hline DoS and DDoS attacks & $\begin{array}{l}\text { There is an intense traffic flow from multiple IP } \\
\text { addresses to the ports of routers and servers }\end{array}$ & $\begin{array}{l}\text { There are violations of the normal functioning of the system, the } \\
\text { availability of data and services is disrupted, which are usually } \\
\text { supplemented by a lack of resources necessary for the network, } \\
\text { operating system or applications }\end{array}$ \\
\hline TCP SYN Flood & $\begin{array}{l}\text { Creating a large number of partially open connections, } \\
\text { increasing the number of SYN packets }\end{array}$ & Violations of the normal functioning of the system \\
\hline Ping of death attacks & Getting too large IP packets & Failure, refusal, freezing and rebooting the system \\
\hline $\begin{array}{l}\text { Tribe Flood Network } \\
\text { (TFN) and Tribe Flood } \\
\text { Network (TFN2K) }\end{array}$ & $\begin{array}{l}\text { Generating packets with spoofed source IP addresses, } \\
\text { dynamically changing the size of packets, IP addresses } \\
\text { and source ports, the appearance in traffic of a large } \\
\text { number of packets per IP address }\end{array}$ & $\begin{array}{l}\text { They are distributed tools, usually launching coordinated DoS } \\
\text { attacks from many sources for one or more purposes }\end{array}$ \\
\hline Stacheldraht & $\begin{array}{l}\text { The appearance of illegal encrypted traffic generation of } \\
\text { packets with spoofed source IP-addresses, dynamically } \\
\text { changing the size of packets }\end{array}$ & $\begin{array}{l}\text { There is an invasion of a large number of systems for their } \\
\text { subsequent use in an attack. }\end{array}$ \\
\hline IP spoofing attacks & $\begin{array}{l}\text { Substitution of source IP addresses with addresses from } \\
\text { trusted zones }\end{array}$ & $\begin{array}{l}\text { An intruder inside or outside the network pretends to be a } \\
\text { computer you can trust }\end{array}$ \\
\hline $\begin{array}{l}\text { "Man-in-the-middle" } \\
\text { attacks }\end{array}$ & $\begin{array}{l}\text { Interception of network packets, routing protocols and } \\
\text { transport protocols, distortion of transmitted data and } \\
\text { inclusion of new information in network sessions }\end{array}$ & $\begin{array}{l}\text { Information theft, hacking of the current session to gain access to } \\
\text { private network resources, traffic analysis - to obtain information } \\
\text { about the network and its users, DoS attacks, distortion of } \\
\text { transmitted data and the inclusion of new information }\end{array}$ \\
\hline Network intelligence & $\begin{array}{l}\text { Requests for the DNS server, scanning for the range of } \\
\text { IP addresses and port scanning. }\end{array}$ & $\begin{array}{l}\text { Attackers can find open ports, examine the characteristics of } \\
\text { applications running on hosts. }\end{array}$ \\
\hline Packet sniffing & $\begin{array}{l}\text { Intercepting packets transmitted over the network in an } \\
\text { open manner (Telnet, FTP, SMTP, POP3, etc.), such as } \\
\text { user names and passwords, switching traffic flows from } \\
\text { one network device to another }\end{array}$ & An attacker can access a system user account. \\
\hline Port forwarding attacks & $\begin{array}{l}\text { Redirects network traffic, dropping in bytes or packets } \\
\text { in a single traffic flow. }\end{array}$ & Passing Illegal traffic via firewall by Intruders \\
\hline
\end{tabular}


A large number of rules in the set of the problem of detecting anomalies may require time resources, there is also the possibility that the specialist who is solving this problem can miss an error or, conversely, calculate for an error that is not so.

To solve the above problems, a method for detecting anomalies in the network traffic is proposed [2-3]. The main differences between the proposed method and existing ones is that the proposed method not only detects anomalies in sets of filtering rules, but simplifies the process of developing sets of filtering rules. The implementation of the proposed method consists of consistently executing the following modules:

- module for interception of the network traffic;

- module for generating formal rules;

- module of parsing network packet.

In Figure 1 is presented the interaction stages of the anomaly detection modules in the network traffic.

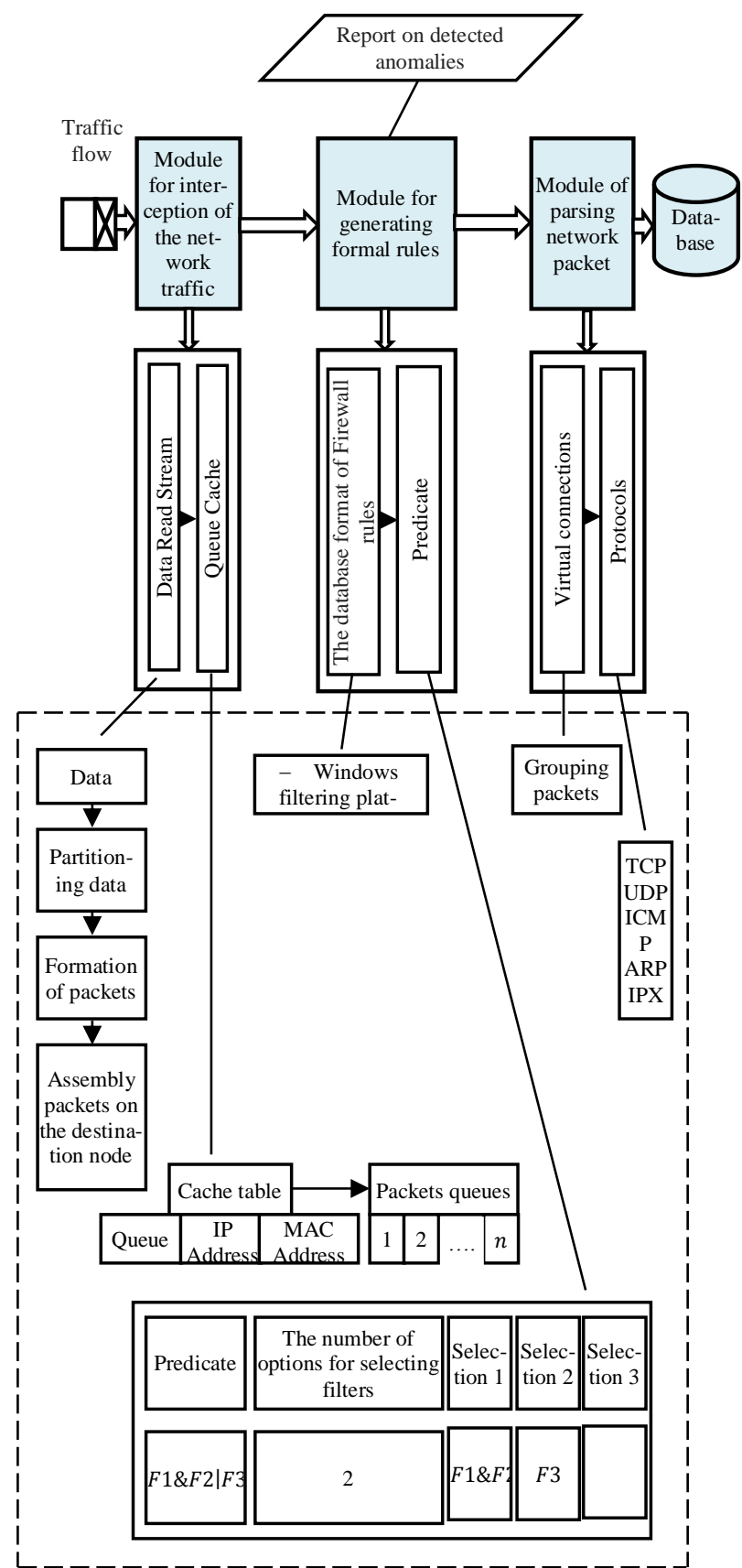

Fig. 1: The Interaction Stages of the Anomaly Detection Modules.
Module for interception of the network traffic. Packets, intercepted by the module for interception of the network traffic are input to the module for generating formal rules. Then the packets are transformed into a module for generating formal rules, that is, rules that are not tied to a particular Firewall model.

Module for generating formal rules. In the process of transformation to the formal form of rules, information is used from the library describing the formats of Firewall rules. The format of the Firewall rules is an XML file containing a scheme for converting formal rules into the format of a particular Firewall. For a number of Firewall that have the binary format of a set of filter rules and do not offer the possibility of exporting / importing it into a text format, the description database may contain not just XML files for conversion schemes, but a library of functions that allow to convert the binary format of the rule set to text and on the contrary. In the event that the required Firewall is not in the list of supported by the system, the user has the ability to create XML files and conversion libraries independently. Then, the filter rules are distributed among a number of filters:

If the rule is permissive, it will be added to each filter.

If the rule is prohibitive, then for a consistent chain of filters, one rule is sufficient and for parallel filter chains, it is sufficient that the rule be applied to one filter from each chain.

If the rule is prohibitive, then it is necessary to determine the forms of the filter predicate.

For filters in one chain, the rule is applied to any of the filters, and here filters are written in the predicate via OR (|).For filters in parallel chains, a rule is applied to each of them and here filters are written in the predicate via AND (\&). The resulting set of formal rules is analyzed and a report on the detected anomalies is then generated.

Module of parsing network packet. In the module of parsing network packet virtual connections are performed. At the end of each of the virtual connections, the source and destination IP addresses, the source and destination ports, the amount of transmitted / received traffic to the database are stored. The only exceptions are ICMP and ARP packets - data about them are stored in the database without aggregation of virtual connections. The database is implemented using SQL-queries, which allows you to take full advantage of all the features and flexibility of the SQL language. By creating the necessary queries to the database, you can get all the necessary information to build a set of filter rules to the point of interception of the analyzed network traffic.

\section{A formal model for protecting network traf- fic from DDOS attacks}

Classification of DDoS-attacks. This is a simplified classification by protocols and by the mechanism of action used to transmit data in computer networks [4-5], the vulnerabilities of which are used by hackers, organizing attacks. In Table 3 is given the classification of DDoS attacks in the computer networks.

In Table 4 is presented DDoS-attacks are possible on each of the seven levels.

Table 3: Classification of DDOS Attacks in the Computer Networks 


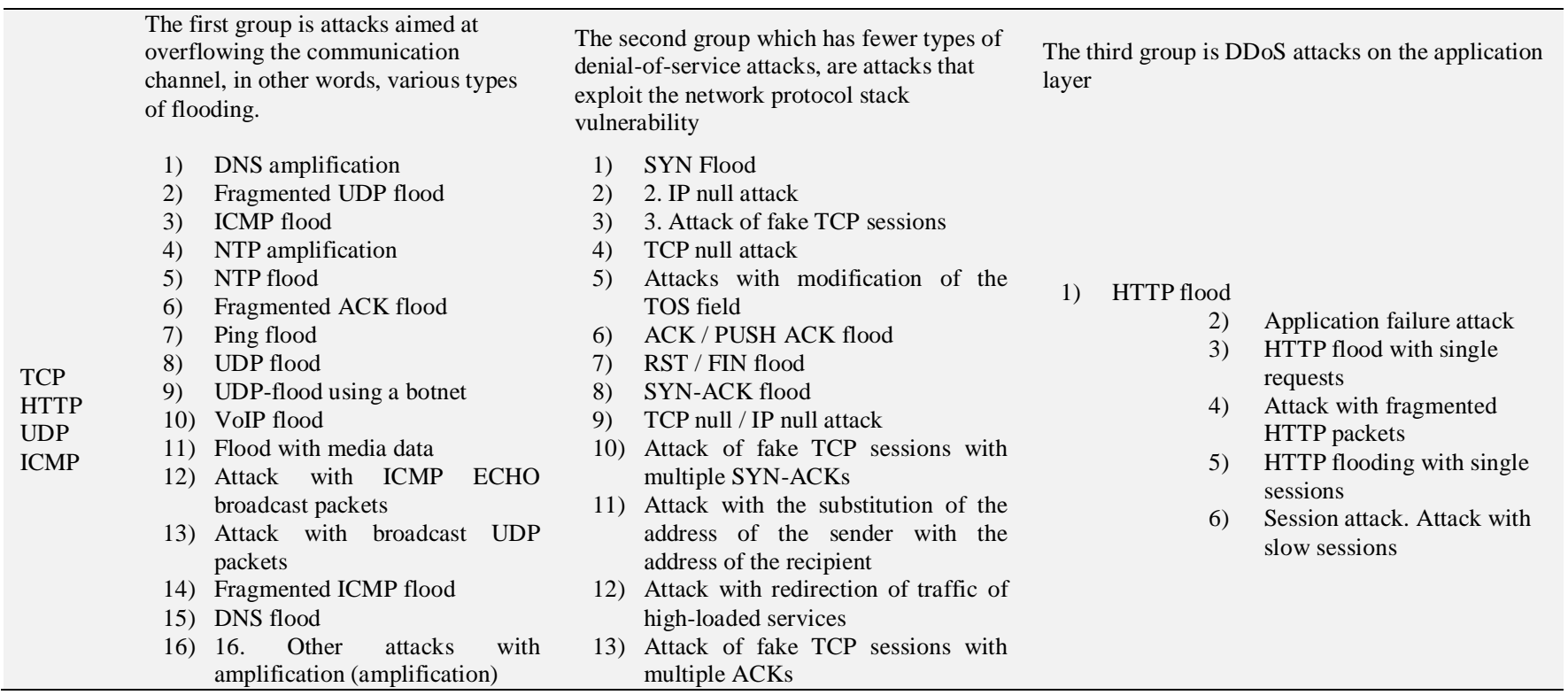

Table 4: Comparative Analysis of Possible Attacks on the OSI Model

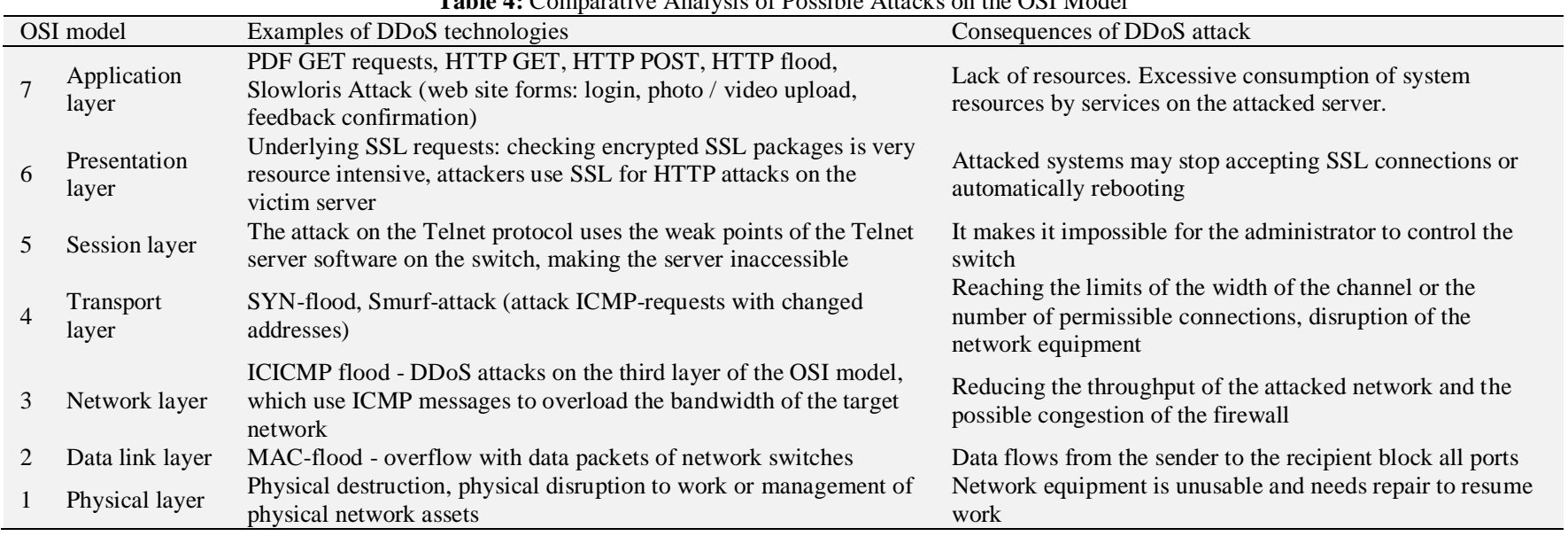

The formal model of information of protection from DDoS attacks is described using formal set-theoretic constructions [6-7].

Let's imagine a model of information protection from DDoS attacks in the form of a tuple:

$M=<I P, O P, H N, H C, I A, U>$

Where

$I P$ - Incoming packets;

$O P$ - Outgoing packets;

$H N$ - A set of nodes (hosts) of the computer network

$H C$ - Set of connections between nodes of the computer network

$I A$ - Scenario of the implementation of the attack;

$U-$ A parameter characterizing the user's actions.

Figure 2 shows a formal model of information of protection from DDoS attacks.

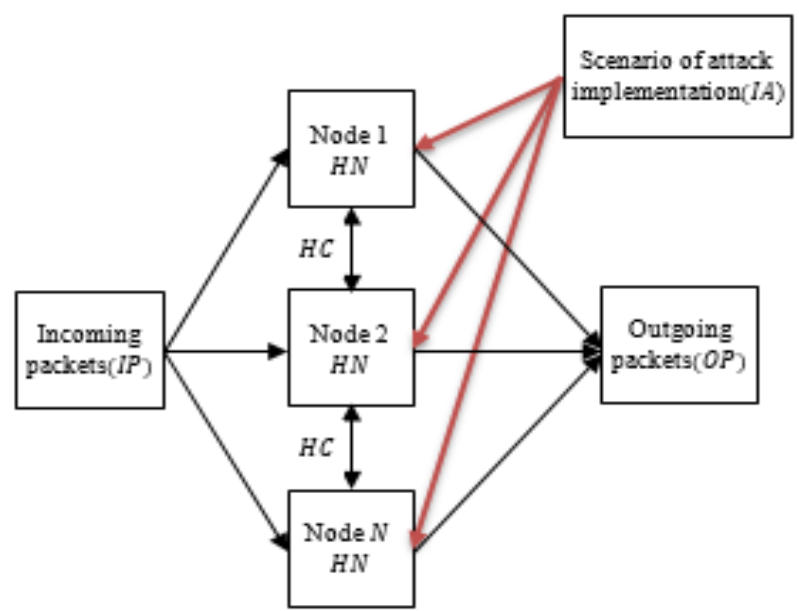

Fig.2: Formal Model of Information Protection from DDOS Attacks.

The set of HN nodes is given in the form of a tuple of elements:

$H N=<$ Equipment, Role, Software, Hardware, Function $>$

Where

Equipment - Multiple types of equipment corresponding to the node of the computer network;

Role - Set of functional roles of the node;

Software - A variety of software components used by the nodes;

Hardware - A set of hardware components used by the nodes; 
Function: Role $\rightarrow$ Software - function that implements mapping of the set of functional roles of a node to a set of software components.

The software and / or hardware component of the software is a protocol that implements a set of rules and allows for the connection and exchange of data between two or more devices included in the network.

The set of HC links between the nodes of the computer network in the context of various protocols is described as follows: it is assumed that the nodes $1,2, \ldots N$ of the network are connected by some protocol if there is at least one non-empty finite sequence with the initial node 1 and the end node 2 through which will be a message.

The scenario for implementing the attack contains:

$I A=<$

$F A_{\text {fuction attack }}, A A_{\text {against attack }}, L A_{\text {legitimate activities }}, W A A_{\text {warning about attack }}$, $R T A_{\text {response to attack }}>$

Where

$F A_{\text {fuction attack }}-$ The functioning of the DDoS attack;

$A A_{\text {against attack }}-$ Deterrence of DDoS attacks and counteracting attacks;

$L A_{\text {legitimate activities }}$ - Legitimate activity of the computer network;

$W A A_{\text {lwarning about attack }}-$ Warning about DDOS attacks

$R T A_{\text {response to attack }}-$ Response to DDOS attacks.

In this case, each intermediate scenario becomes the object of subsequent decomposition.

Scenarios $F A_{\text {fuction attack }}$ contain sub-scenarios for the spread of the DDoS attack, its management and the implementation of attacks.

Scenarios $A A_{\text {against attack }}$ contain sub-scenarios to counteract the spread of DDoS attacks, counteracting its management and countering the implementation of attacks.

Scenarios $L A_{\text {legitimate activities }}$ are designed to generate legitimate traffic patterns.

Scenarios $W A A_{\text {lwarning about attack }}$ are designed to mitigate the consequences of an attack on a victim.

Scenarios $R T A_{\text {response to attack }}$ are designed to detect and respond to DDoS attacks.

\section{Conclusion}

Because of the measuring network traffic experiment, it was revealed that for packet interception and traffic dump analysis it is necessary to consider the size, length and delay of packets and decrease the lifetime of the virtual TCP connection for each packet. With the analysis of the traffic dump, a method for detecting anomalies in the network traffic has been developed, which makes it possible to reduce information security risks with improperly configured rules and reduce the number of network anomalies of filtering rules in the computer networks. Based on the method for detecting anomalies in the network traffic, a formal model for protecting network traffic from DDoS attacks is proposed, which allows more efficient protection of networks from unauthorized traffic.

\section{References}

[1] LinY-D., LuCh-N., LaiY- Ch., etal. Application classification using packet size distribution and port association // J. Network Computer Appl. 2009. V.32. - P.1023-1030.

[2] Gulomov Sh.R. Rakhmanova G.S., Boymurodov B.E. Ensuring Secure Info-Communication Networks Based on the Special Filtering Mode. International Journal of Engineering Innovation \& Research. Volume 5, Issue 1, 2016, ISSN: 2277 - 5668, India, -P.16-23.

[3] William Stallings. Network security essentials: Applications and Standards Fourth edition. Prentice Hall, USA, 2011. - P.417.
[4] Behrouz A. Forouzan. Data Communications and Networking.5th Edition / McGraw-Hill Forouzan series, New-York USA, 2007. P.1134.

[5] William Stallings. Data and Computer Communications (10th Edition). International Edition, 2013. - P.912.

[6] D. van Dalen: Logic and Structure (fourth extended ed. Revised). (Springer Verlag, Berlin, 2008)

[7] M. Rathjen: Metamathematical Properties of Intuitionistic Set Theories with Choice Principles. In: S.B. Cooper, B.Lowe, A.Sorbi (eds.): New Computational Paradigms: Changing Conceptions of What is Computable (Springer, New York, 2008) P.287-312. https://doi.org/10.1007/978-0-387-68546-5_13. 